\title{
Morris screening for FMECA of valve failure modes on offshore gas reinjection
}

\author{
Nielsen, Emil Krabbe; Frutiger, Jerome; Sin, Gürkan
}

Published in:

Proceedings of the 29th European Symposium on Computer Aided Process Engineering

Publication date:

2019

Document Version

Peer reviewed version

Link back to DTU Orbit

Citation (APA):

Nielsen, E. K., Frutiger, J., \& Sin, G. (2019). Morris screening for FMECA of valve failure modes on offshore gas reinjection. In K. Anton, E. Zondervan, R. Lakerveld, \& L. Özkan (Eds.), Proceedings of the 29th European Symposium on Computer Aided Process Engineering (pp. 1315-1320). Elsevier. Computer Aided Chemical Engineering Vol. 46

\section{General rights}

Copyright and moral rights for the publications made accessible in the public portal are retained by the authors and/or other copyright owners and it is a condition of accessing publications that users recognise and abide by the legal requirements associated with these rights.

- Users may download and print one copy of any publication from the public portal for the purpose of private study or research.

- You may not further distribute the material or use it for any profit-making activity or commercial gain

- You may freely distribute the URL identifying the publication in the public portal 


\title{
Morris screening for FMECA of valve failure modes on offshore gas reinjection
}

\author{
Emil Krabbe Nielsen ${ }^{\mathrm{a}, \mathrm{b}}$, Jérôme Frutiger ${ }^{\mathrm{b}}$ and Gürkan $\operatorname{Sin}^{\mathrm{b}}{ }^{*}$ \\ a Department of Electrical Engineering, Technical University of Denmark, Elektrovej 326, Kgs. \\ Lyngby 2800, Denmark \\ ${ }^{\mathrm{b}}$ Department of Chemical Engineering, Technical University of Denmark, Sltofts Plads 227, Kgs. \\ Lyngby 2800, Denmark \\ ekrani@elektro.dtu.dk
}

\begin{abstract}
FMECA is commonly used as a tool for assessing the consequences of different failure modes of a component and the criticality of the consequences. Traditionally failure modes are generated using a one-factor-at-a-time method. In this study, we propose extensions to the FMECA procedure in which failure modes are sampled using statistical sampling techniques, and their effects are evaluated under a wide range of operating conditions. Morris's efficient sampling technique is used for generating failure mode effect analysis scenarios. The scenarios are evaluated in a KSpice simulator with a model of an offshore gas reinjection system in the Danish North Sea. The impact of the failure modes on the process performance and process safety is evaluated under varying process conditions. The extended methodology enables fast screening of the effects of the failure mode under different realistic process conditions. This provides a more comprehensive and global assessment of the consequences for process safety and reliability in the chemical industry.
\end{abstract}

Keywords: Failure Mode Effect and Criticality Analysis, Morris screening, Gas reinjection

\section{Introduction}

For safety-critical systems, tools like FMECA and HAZOP are important in reliability analysis as well as Quantitative Risk Assessment (QRA) studies to ensure an inherently safe design and safe operation of the system. For modern chemical process plants, a model is a requirement in the design phase. This model should be exploited, not just to ensure mass, energy and momentum balances of the system, and to optimize productivity, but also to ensure the safety of the system. In previous studies simulations are conventionally performed according to a single defined operational setpoint to evaluate failure modes Enemark-Rasmussen et al. (2012). However as economical experimental designs are available Morris (1991) and computational power increases, such evaluations should be carried out by rigorously investigating the influence of failure modes under a range of potential variation of the process conditions.

In this work we propose a quantitative approach to analyse the effects of failure modes of a control valve under a range of process conditions. We have identified four failure modes of control valves on offshore oil and gas platforms that will be simulated under varying process conditions. The method is more time consuming and computationally expensive than conventional approaches, especially as the number of process conditions, components and failure modes increases with system complexity. To avoid an excessively time consuming method, the process conditions, and the failure modes are sampled by using a random and economically efficient sampling method: 
Morris screening. Morris screening is a discrete sampling method, and allows for evaluation of different and completely unrelated failure modes in one study.

\section{Process system}

The approach is applied to an offshore gas reinjection system of Mærsk, that has been modelled in $\mathrm{K}$-Spice based on design and operation data in Enemark-Rasmussen et al. (2012). The purpose of the gas reinjection system is to maintain the well pressure for enhanced oil recovery. The feed of gas from upstream is controlled by a control valve. In the reinjection system the gas is first cooled by a heat exchanger, supplied with sea water. The cooling rate is controlled by a control valve, at the outlet of the heat exchanger. Next, the gas enters the scrubber to avoid vapour in the gas being reinjected to the well. Production of liquids from the scrubber is undesired, however a control valve releases the liquid to a flare, if the level becomes too high. A compressor sucks the gas from the scrubber, after which the gas is either reinjected to the wells or recycled. The gas is recycled to avoid the compressor from surging. Surging is undesirable, however so is recycling the gas as it limits the productivity. An anti-surge controller controls the setpoint of four control valves, one for gas feed, two for gas recycling and one for gas reinjection, to ensure a steady flow through the compressor. The modelled system in K-Spice is shown in Figure 1.

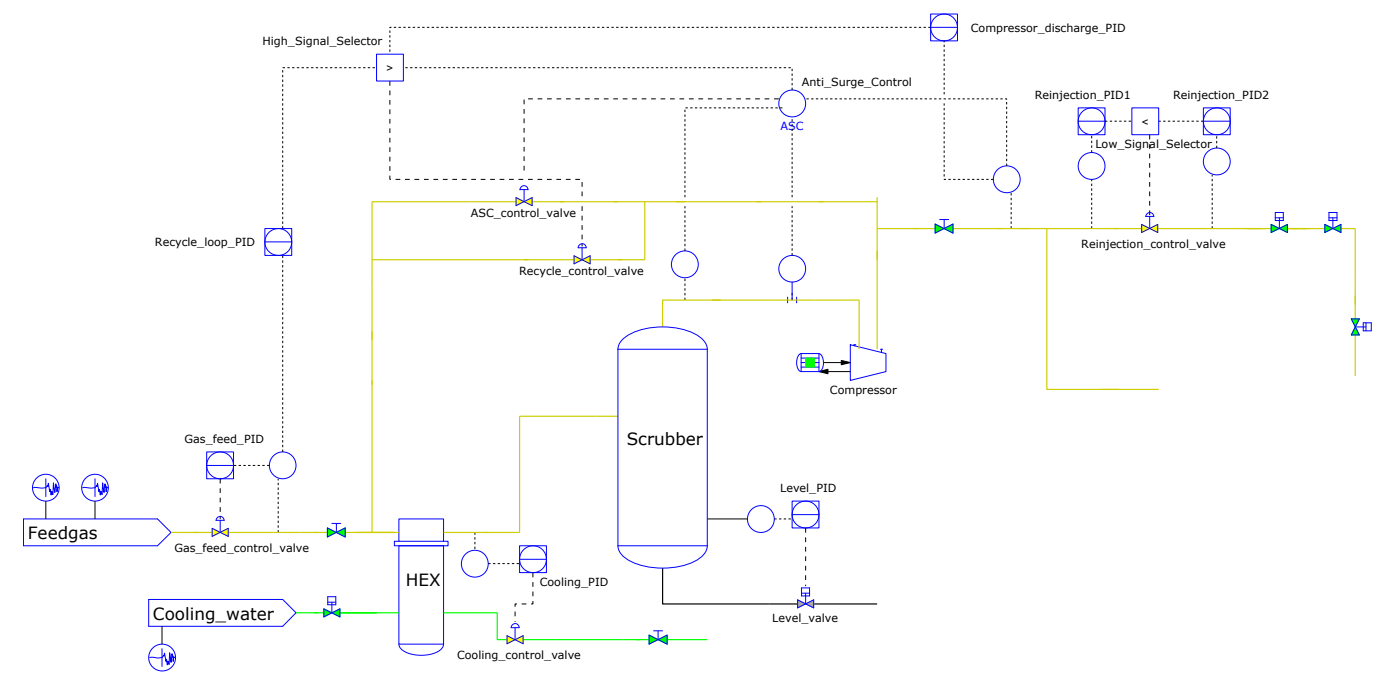

Figure 1: Offshore gas reinjection system.

Various different failure modes have previously been simulated in K-spice for this system EnemarkRasmussen et al. (2012). However common for all simulations was the use of only a single set of process conditions according to design specifications. The process conditions before and after introducing the failure mode was used to calculate the sensitivity of the process to assess the criticality of each failure mode using a one-factor-at-a-time approach. In this study, we use a global sampling based approach to assess the criticality of the consequences, and focus the study on four failure modes of one control valve evaluated under a range of process conditions.

The effort to document the occurrence of failure modes for valves, pumps etc. is extensive in the oil and gas industry, however the level of detail on reported failure modes is very sparse Management (2002); Peters and Sharma (2003). In this study, the valve opening $P$, and the valve opening and closing time $t_{\text {open }}$ and $t_{\text {close }}$ are used for implementing four different failure modes for the gas feed control valve. When implementing the failure mode, the manipulated parameter is fixed for the remaining simulation from $t_{f m}$ to $t_{\text {end }}$. Apart from the failure modes, the sampled initial process 
conditions are the temperature $T_{\text {gas }}$ and the pressure $P_{\text {gas }}$ of the feed gas, and additionally the feed temperature $T_{\text {cool }}$ of the sea water used for cooling.

The values of the sampled initial conditions is determined by a discrete cumulative distribution function (CDF). The variation in percentage for the conditions are as follows: $T_{g a s}=5 \%, P_{\text {gas }}=$ $4 \%$ and $T_{\text {cool }}=4 \%$. As described in Sin et al. (2009), it can be used to determine the minimum and maximum values of a uniform distribution discretized into $p=4$ levels, around a mean or nominal value of operation. The nominal operation is defined as: $T_{\text {gas }}=44.5^{\circ} \mathrm{C}, P_{\text {gas }}=121 \mathrm{bar}$ and $T_{\text {cool }}=30^{\circ} \mathrm{C}$. Gaussian noise is added to the process conditions as measurement noise and varies during the simulation. The standard deviation $\sigma_{\text {noise }}$ in Table 3 defines the normal distribution of the noise, around the values sampled by Morris screening for $\theta_{1 i}, \theta_{2 i}$ and $\theta_{3 i}$ as mean. The noise is added as part of the simulation in K-Spice, and is sampled randomly by $\mathrm{K}$-spice.

\section{Procedure}

First a set of $k$ process conditions including the failure mode is sampled as input $\theta_{i}=\left[\theta_{1 i}, \theta_{2 i} \ldots \theta_{k i}\right]$ for the simulation for $i=1,2 \ldots n$ simulations. The $n$ number of sampled sets are then simulated in K-Spice. All simulations start with a sampled set of initial process conditions $\theta_{1 i}, \theta_{2 i}$ and $\theta_{3 i}$ at time $t_{\text {start }}=0 \mathrm{~s}$. These conditions are used throughout the entire simulation. To allow the system to stabilize under the set of process conditions specified by the sampling, the simulation is run for 1200 seconds. At time $t_{f m}=1200 \mathrm{~s}$ the failure mode (FM) $\theta_{4}$ is introduced. Next, the simulation is run for 7200 seconds until $t_{\text {end }}=8400 \mathrm{~s}$ after which the simulation is stopped, and the simulation output for every simulation $Y_{i j}=\left[y_{1 i j}, y_{2 i j} \ldots y_{m i j}\right]$ for $j=\left[10,20 \ldots t_{\text {end }}\right]$ is recorded by sampling every ten seconds. Here, $j$ denotes a point in the time series of the $m$ th output signal from the $i$ th simulation. The steps, inputs and outputs of the procedure is shown in Table 3.

\begin{tabular}{llll}
\hline$\#$ & Step & Input & Output \\
\hline 1 & Morris screening & $\mathrm{r}=40, \mathrm{k}=4, \mathrm{p}=4, \Delta=2 / 3, \mathrm{CDF}$ & $\theta_{i}$ \\
2 & Load initial conditions in simulation $i$ & $\theta_{1 i}, \theta_{2 i}, \theta_{3 i}$ \\
3 & Run simulation $i$ untill $t_{f m}=1200 \mathrm{~s}$ & $\sigma_{\text {Noise }}=[5,2.5,2.5]$ & \\
4 & Introduce failure mode & $\theta_{4}$ & \\
5 & Run simulation $i$ untill $t_{\text {end }}=8400 \mathrm{~s}$ & $\theta_{i j}, Y_{i j}$ & $E E_{i}$ \\
6 & Sensitivity analysis & $\theta$ & $Y$ \\
\hline
\end{tabular}

Table 1: Procedure for sampling, simulating and analysing.

\section{Experiment and sample design}

Morris screening is a global sensitivity analysis method that employs a one-factor-at-a-time (OAT) approach for producing experimental designs. OAT analysis is performed for a number of randomly sampled nominal values in design space. The analysis is based on the mean and standard deviation of the elementary effects, $E E_{i}$ for input $i$ to determine how sensitive the outputs are to changes in the inputs $i=1,2, \ldots$. For these experiments $k=4$ input parameters are sampled at $p=4$ levels. Typically, the number of repetitions needed for Morris screening is in the range of $r=[10,50]$ Sin et al. (2009), and based on the required number of elementary effects $F_{i}$ to be calculated. For this study $r=40$ is used, however only five elementary effects are shown. Based on this, the number of samples are $n=r(k+1)=40(4+1)=200$ Sin et al. (2009). The elementary effects are calculated based on changes to the inputs, defined by the step size $\Delta=p /[2(p-1)]=4 /[2(4-1)]=2 / 3$ for a uniform distribution of the sampling space. These steps are used for performing local sensitivity analysis, however when averaging the mean of the elementary effects, the method can be used in a global context. The sampled probabilities in unit hyperspace [ $\left[\begin{array}{ll}1\end{array}\right]$ have been converted to real values by using their respective inverse discrete and 
cumulative probability distribution function. The values of the resulting four levels are shown in table 4.

\begin{tabular}{ccccl}
\hline Level & $T_{\text {gas }}, \theta_{1}$ & $P_{\text {gas }}, \theta_{2}$ & $T_{\text {cool }}, \theta_{3}$ & Failure mode $(\mathrm{FM}), \theta_{4}$ \\
\hline 1 & $28.6^{\circ} \mathrm{C}$ & $116.2 \mathrm{bar}$ & $17.9^{\circ} \mathrm{C}$ & Valve seized at current position $P$ \\
2 & $39.2^{\circ} \mathrm{C}$ & $119.4 \mathrm{bar}$ & $26.0^{\circ} \mathrm{C}$ & Valve fail open at $P=1 \%$ \\
3 & $49.8^{\circ} \mathrm{C}$ & $122.6 \mathrm{bar}$ & $34.0^{\circ} \mathrm{C}$ & Valve fail close at $P=99 \%$ \\
4 & $60.4^{\circ} \mathrm{C}$ & $125.8 \mathrm{bar}$ & $42.1^{\circ} \mathrm{C}$ & Stiction $3 t_{\text {close }} \& 3 t_{\text {open }}$ \\
\hline
\end{tabular}

Table 2: Design space.

\section{Results}

For each simulation $i$, the output $Y_{i j}$ is a time series with $j$ samples in time, corresponding to the sampled input $\theta_{i}$. A selection of the process signals from the simulations are shown in Figure 2. The average of all 200 samples for each time step $j$ is plotted for each output, along with a lower and upper safety limit. The limits are an average of the 200 process signals for each output from time $t_{\text {start }}$ to $t_{f m}$, multiplied by $\pm 25 \%$. In general the control system is capable of maintaining the temperature close to nominal operating conditions as can be seen on plot 1-5 in Figure 2. However, under some conditions, the temperature exceeds the upper limit at the gas outlet, and in the recycle loop. The plots 6-9 shows a stable pressure under the majority of process conditions, although it drops below the lower limit to nearly 0 bar under some conditions. This does however not pose a safety risk. Plot 10 shows that the amount of recycled gas varies a lot between 0 and $200 \mathrm{~kg} / \mathrm{h}$, and under some specific conditions the recyled amount approaches $300 \mathrm{~kg} / \mathrm{h}$. In general the productivity is stable, but it drops close to $0 \mathrm{~kg} / \mathrm{h}$ under some conditions. The surge rate, defined as $\frac{\int \text { Timesurgingdt }[s]}{\text { Time }[s]}$, varies a lot initially, as expected, and in time splits up into four dinstinct bands, for which two of them increases over time, one remains constant and one decreases over time.
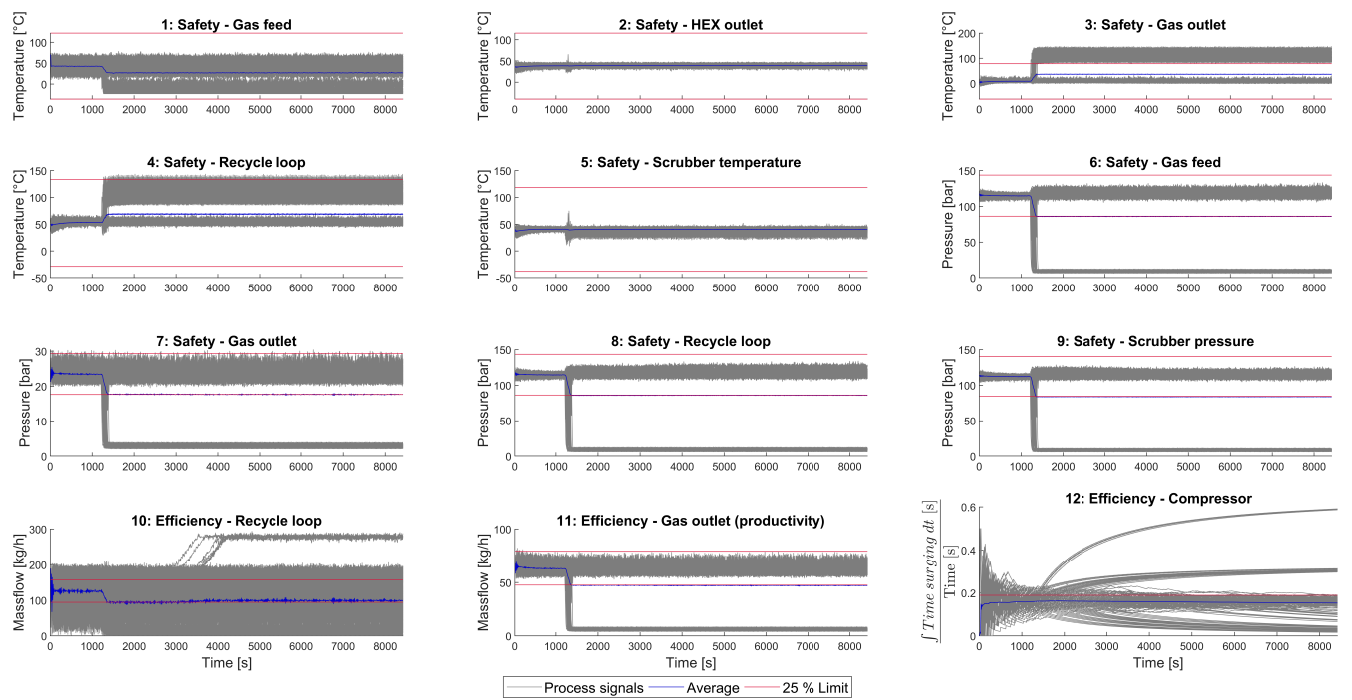

Figure 2: A selection of process signals for output $Y_{i j}$ from the $n=200$ samples.

The plots shown in Figure 3 of each output as a function of each input shows that failure mode two is the sole cause of the high temperatures for the gas outlet and recycle loop, independent 
of the process conditions. The plot also shows, that failure mode two is the sole reason for the pressure drop in the gas reinjection system, as only very little new gas is supplied. On the contrary, failure mode four consistently produces high pressure with almost no variation. The amount of recycled gas drops close to $0 \mathrm{~kg} / \mathrm{h}$ for failure mode two as only a small amount of gas is supplied to the system. Failure mode three causes the high amount of recycled gas for a gas feed pressure of 125.8 bar. As previously mentioned, the productivity is stable, which is true for all process conditions, except those including failure mode two. Failure mode two results in three bands of surging, resulting in both the lowest and highest amount of time surged in total. The plots do however not show any unique set of conditions producing these bands. The variation in the amount of time surged is very low for both failure mode one and four as opposed to two and three.

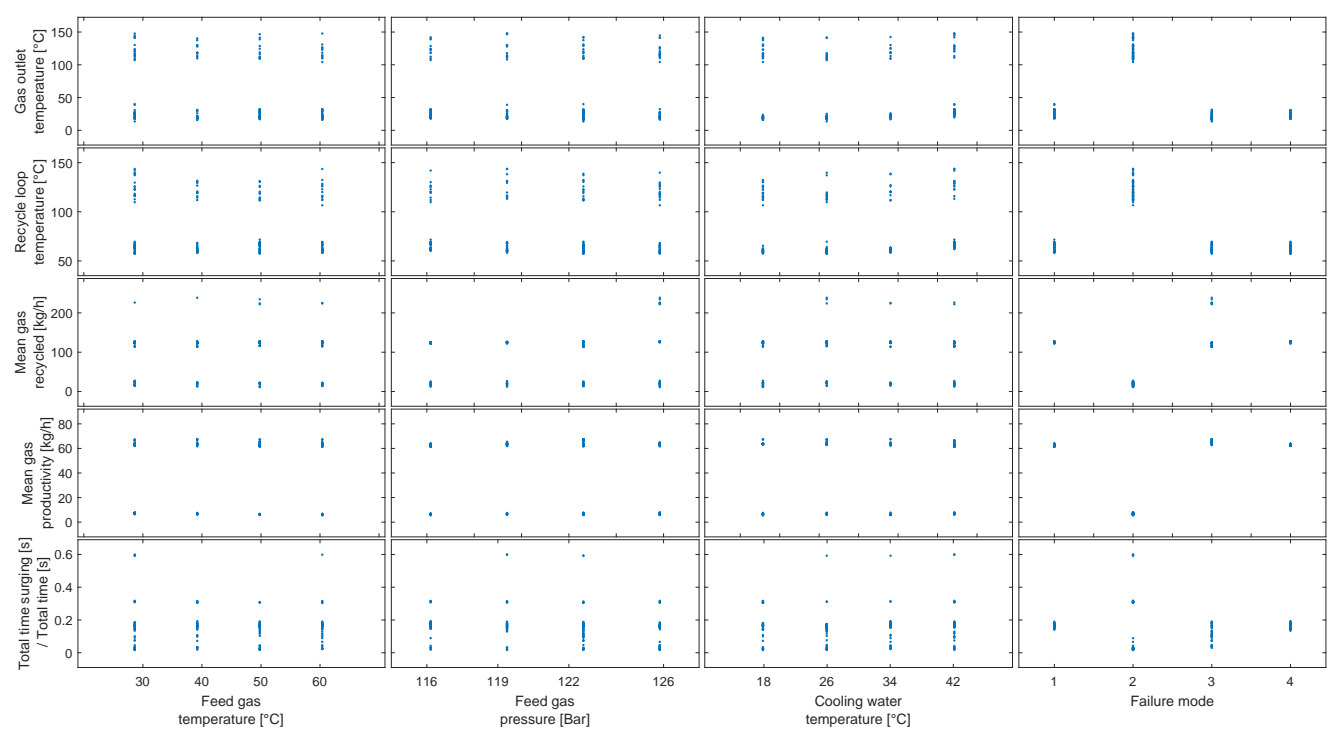

Figure 3: Matrix plot of model output $Y_{i}$ as a function of model input $\theta_{i}$.

The most significant elementary effect has the highest average, and a high standard deviation is an indication of interactions or non-linearity Morris (1991). The elementary effects for some of the critical outputs are shown in Figure 4. The wedge in the figure can be used as an indication of significance, and is calculated as the mean of samples of the elementary effects: $\hat{d}_{i}= \pm 2 S E M_{i}$. The standard error of mean, $S E M_{i}$ is calculated as the variance $S_{i}$ of the elementary effects of input sample $i$ divided by the square root of repetitions $r: S E M_{i}=\frac{S_{i}}{\sqrt{r}}$. If an elementary effect is outside of the wedge it is considered significant, and insignificant if inside Morris (1991).

A change in failure mode can be considered as the most influential input parameter on the gas outlet temperature, recycle loop temperature, amount of gas recycled and the gas productivity, but shows no significance on the amount of time surged. This result for the total surge rate contradicts the observations from Figure 3. The values in Figure 4 are averaged elementary effects based on all changes to the respective input parameter. This can lead to ambiguous results and for this reason these results cannot be interpreted alone. The gas feed pressure change should also be considered significant for the increase in gas outlet temperature, and a change in temperature of the cooling feed is significant for an increase in temperature in the recycle loop. The mean of the total surge rate shown in Fig. 4 indicates no significant inputs, as the standard error of the mean $S E M_{i}$ is quite large due to the high variance $S_{i}$ of elementary effects. The estimated mean $\mu^{*}$ of the distribution of absolute values of the elementary effects is however useful for Type II errors when the variance is both negative and positive Campolongo et al. (2007). It reveals that the failure mode is the most significant parameter for the variation of the surge rate as $\mu^{*}=5.70$ for FM, $\mu^{*}=5.51$ for $T_{\text {cool }}$, 

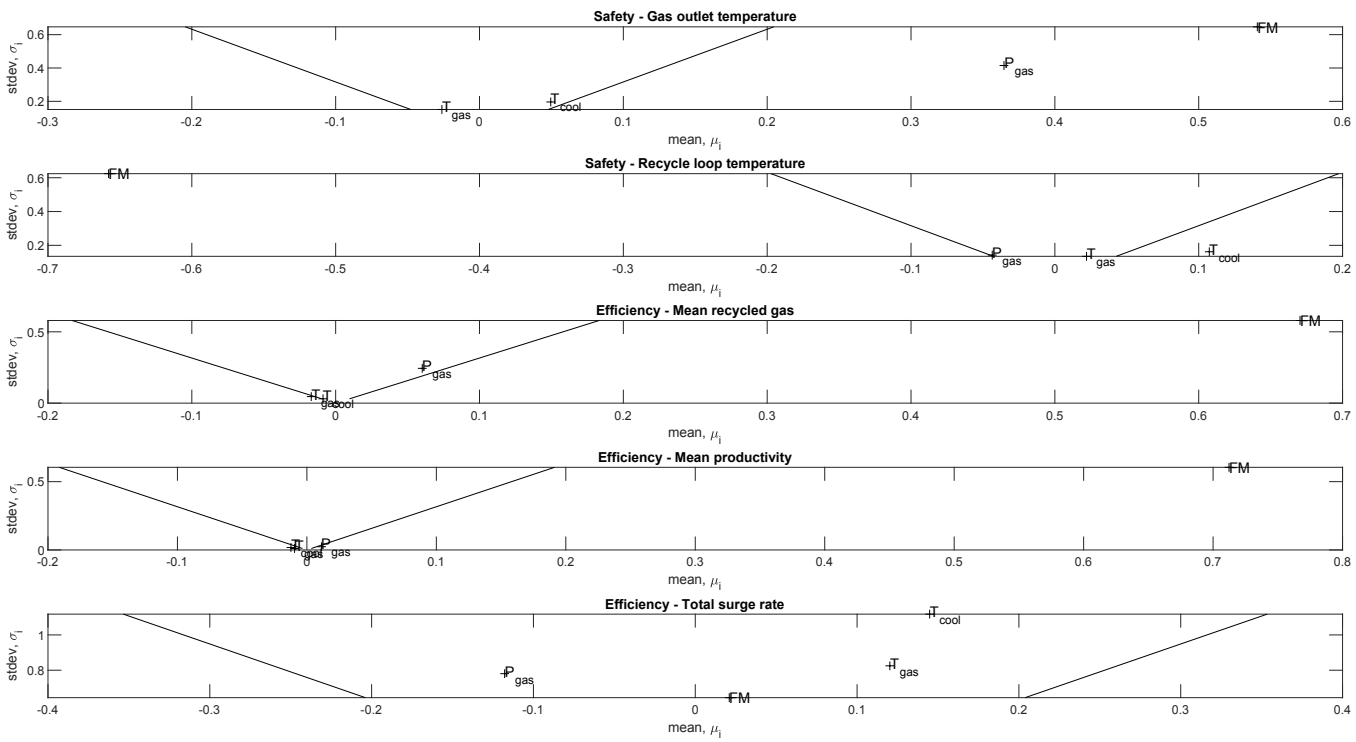

Figure 4: Elementary effects of outputs.

$\mu^{*}=4.02$ for $T_{\text {gas }}$ and $\mu^{*}=3.98$ for $P_{\text {gas }}$.

In general, the standard deviation of the elementary effects of the failure mode is quite large for all outputs except for the surge rate. However as this input parameter is not continuous, but merely four completely different parameters in itself, a high standard deviation is expected and should thus be either interpreted with caution or disregarded.

\section{Conclusion}

Morris screening has been used for analysing failure modes of a valve under varying process conditions in simulations of an offshore gas reinjection system. The analysis showed that only the temperature at the gas outlet and in the recycle loop could compromise the safety, caused by the gas feed valve closing. In general the results showed that within the studied range of variation the process conditions are insignificant, and that the effect of the failure modes is independent of the process conditions. The efficiency of the plant was also examined, and in general the productivity was stable, except for when the valve closes, and the gas feed is shut off. Additionally, the closed valve impacts the amount of time which the compressor surged both negatively and positively. In case of an open valve, the recycling rates increased, but only under high pressure.

\section{References}

F. Campolongo, J. Cariboni, A. Saltelli, 2007. An effective screening design for sensitivity analysis of large models. Environmental modelling \& software 22 (10), 1509-1518.

R. Enemark-Rasmussen, D. Cameron, P. B. Angelo, G. Sin, 2012. A simulation based engineering method to support hazop studies. In: Computer aided chemical engineering. Vol. 31. Elsevier, pp. 1271-1275.

S. I. Management, 2002. OREDA: Offshore Reliability Data Handbook, 4th Edition. OREDA Participants.

M. D. Morris, 1991. Factorial sampling plans for preliminary computational experiments. Technometrics 33 (2), 161174.

J. Peters, R. Sharma, 2003. Assessment of valve failures in the offshore oil \& gas sector. Health and Safety Executive.

G. Sin, K. V. Gernaey, A. E. Lantz, 2009. Good modeling practice for pat applications: Propagation of input uncertainty and sensitivity analysis. Biotechnology progress 25 (4), 1043-1053. 